\title{
Erratum to: Development of a Multiplex- PCR probe system for the proper identification of Klebsiella variicola
}

Ulises Garza-Ramos ${ }^{1 *}$, Jesús Silva-Sánchez ${ }^{1}$, Esperanza Martínez-Romero², Perla Tinoco ${ }^{1}$, Marisol Pina-Gonzales ${ }^{1}$, Humberto Barrios ${ }^{1}$, Jesús Martínez-Barnetche ${ }^{3}$, Rosa Elena Gómez-Barreto ${ }^{3}$ and Juan Tellez-Sosa ${ }^{3}$

\section{Erratum}

The original version of this article [1] unfortunately contained a mistake in Table 1 . The primer sequence which corresponds to $\mathrm{KmtnC}-\mathrm{R}$ in the table 1 was provided incorrectly. The correct nucleotide sequence is:

KmtnC-R:GCATGGCCCAGGTGTTCAG

An updated version of Table 1 has been provided below.

\begin{abstract}
Author details
'Departamento de Diagnóstico Epidemiológico, Av. Universidad \# 655, Col. Sta. Ma. Ahuacatitlán, C.P. 62100 Cuernavaca, Morelos, Mexico. ${ }^{2}$ Centro de Ciencias Genómicas (CCG), Universidad Nacional Autónoma de México (UNAM), Cuernavaca, Morelos, Mexico. ${ }^{3}$ Instituto Nacional de Salud Pública (INSP), Centro de Investigación Sobre Enfermedades Infecciosas (CISEI),

Departamento de Inmunología, Cuernavaca, México.
\end{abstract}

Received: 19 February 2016 Accepted: 2 March 2016

Published online: 16 March 2016

\section{References}

1. Garza-Ramos et al. BMC Microbiology (2015) 15:64. doi:10.1186/s12866-015-0396-6.

\footnotetext{
* Correspondence: ulises.garza@insp.mx

${ }^{1}$ Departamento de Diagnóstico Epidemiológico, Av. Universidad \# 655, Col.

Sta. Ma. Ahuacatitlán, C.P. 62100 Cuernavaca, Morelos, Mexico
} 
Table 1 Amplification conditions, oligonucleotide combinations, sequence and amplification fragment of multiplex-PCR for K. variicola identification

\begin{tabular}{|c|c|c|c|c|c|c|c|}
\hline \multirow{2}{*}{$\begin{array}{l}\text { Amplification } \\
\text { conditions }^{a}\end{array}$} & \multirow{2}{*}{$\begin{array}{l}\text { Name of } \\
\text { combination } \\
\text { primers }\end{array}$} & \multicolumn{6}{|c|}{ Shared unique genes, oligonucleotides and sequence $\left(5^{\prime}-3^{\prime}\right)$ of each bacterial specie } \\
\hline & & K. pneumoniae & $\begin{array}{l}\text { Amplification } \\
\text { fragment (bp) }\end{array}$ & K. variicola & $\begin{array}{l}\text { Amplification } \\
\text { fragment (bp) }\end{array}$ & Klebsiella spp. & $\begin{array}{l}\text { Amplification } \\
\text { fragment (bp) }\end{array}$ \\
\hline & & phosphohydrolase & & phosphoglycerate mutase & & $\begin{array}{l}\text { phosphopentane phosphatase } \\
\text { (mtnC) }\end{array}$ & \\
\hline \multirow[t]{3}{*}{1} & M-PCR-1 & $\begin{array}{l}\text { KP888-F: } \\
\text { AAGCAAGCCAGAACAGAAAG }\end{array}$ & 888 & $\begin{array}{l}\text { KV770-F: TCCCGAGGTTCA } \\
\text { CATTTCC }\end{array}$ & 449 & $\begin{array}{l}\text { KmtnC-F: } \\
\text { CCGCCGACCTTATCACTAC }\end{array}$ & 340 \\
\hline & & $\begin{array}{l}\text { KP888-R: } \\
\text { ACTTCGGTTTTATCCAGGTC }\end{array}$ & & $\begin{array}{l}\text { KV770-R: AGCGGGTGAAC } \\
\text { GTCGATAC }\end{array}$ & & $\begin{array}{l}\text { KmtnC-R: } \\
\text { GCATGGCCCAGGTGTTCAG }\end{array}$ & \\
\hline & & transferase (yphG) & & $\mathrm{N}$-acetyltransferase & & $\begin{array}{l}\text { phosphopentane phosphatase } \\
\text { (mtnC) }\end{array}$ & \\
\hline \multirow[t]{3}{*}{1} & M-PCR-2 & $\begin{array}{l}\text { KP878-F: } \\
\text { ACCGATAACCAGCCTGACTT }\end{array}$ & 878 & $\begin{array}{l}\text { KV1615-F: ACACAACATTT } \\
\text { CAGGCGGCT }\end{array}$ & 499 & $\begin{array}{l}\text { KmtnC-F: } \\
\text { CCGCCGACCTTATCACTAC }\end{array}$ & 340 \\
\hline & & $\begin{array}{l}\text { KP878-R: CTTTCTTCTGCCCA } \\
\text { CTGTTG }\end{array}$ & & $\begin{array}{l}\text { KV1615-R: GGGCGTGGCTT } \\
\text { TाTTCATCG }\end{array}$ & & $\begin{array}{l}\text { KmtnC-R: } \\
\text { GCATGGCCCAGGTGTTCAG }\end{array}$ & \\
\hline & & phosphohydrolase & & thiopurine S-methyltransferase & & $\begin{array}{l}\text { phosphopentane phosphatase } \\
\text { (mtnC) }\end{array}$ & \\
\hline \multirow[t]{2}{*}{2} & M-PCR-3 & $\begin{array}{l}\text { KP888-F: AAGCAAGCCAGA } \\
\text { ACAGAAAG }\end{array}$ & 888 & $\begin{array}{l}\text { KV1000-F: CTGGGATGTGG } \\
\text { CAATGGTG }\end{array}$ & 438 & $\begin{array}{l}\text { KmtnC-F: } \\
\text { CCGCCGACCTTATCACTAC }\end{array}$ & 340 \\
\hline & & $\begin{array}{l}\text { KP888-R: ACTTCGGTTTTAT } \\
\text { CCAGGTC }\end{array}$ & & $\begin{array}{l}\text { KV1000-F: AAACTGCGCCT } \\
\text { GCTGTATC }\end{array}$ & & $\begin{array}{l}\text { KmtnC-R: } \\
\text { GCATGGCCCAGGTGTTCAG }\end{array}$ & \\
\hline
\end{tabular}

${ }^{a}$ Multiplex-PCR conditions used under the oligonucleotides combinations. 1: 5pmol/reaction of $K$. variicola and Klebsiella spp, 25pmol/reaction of $K$. pneumoniae; $2: 25$ pmol/reaction of $K$. pneumoniae, 5 pmol/reaction of K. variicola and $1 \mathrm{pmol} /$ reaction of Klebsiella spp 